\title{
Design Research of the Insurance Business Management System Facing Mobile Terminals
}

\author{
SU Wei ${ }^{1, a}$ \\ ${ }^{1}$ Qinghai university of finance and economics college, Qinghai Xining 810001,China \\ asuweiqh@163.com
}

Keywords: Computer-aided Testing; English; Real- time Testing; Online examination

\begin{abstract}
Insurance plays a very important means of economic compensation in the path of economic development and construction in our country. In the aspect of the comprehensive development of society it has played a positive effect. At the same time, the insurance industry itself also made great development. In this development trend, the insurance agent and insurance broker company arises at the historic moment, and gradually took on more and more insurance product sales business. But with the development of the insurance market, the competition degree is intensifying, the insurance business growing, so it is becoming more and higher for insurance brokerage company informatization construction demands and expectations, including the requirement of business management mode, the requirement of application software function and the information system maintenance requirements. In this case, the establishment of a comprehensive information management platform becomes the inevitable demand of the mainstream insurance brokerage company.
\end{abstract}

\section{Functional requirements analysis of mobile business system}

As a general platform, mobile application server front desk support different terminals, including different types like Phone, PAD and different operating systems like iOS and androidmobile terminals. Background supports different enterprise management system, such as ERP, collaborative office, CRM, SCM, etc. The same APP support multiple service, a service support connect to multiple backend systems. According to the functional requirements analysis, the mobile application server management this paper needed to implement includes super administrator, equipment management, password policy management, log management and MA system. [1]It is from two aspects of the server and client, the five major functional modules and interface design achieve together, and bearing is the function of data transmission interface. In addition, from the main function of the system the enterprise information system this article abstracts mobile client business types, including mobile, mobile warning message of examination and approval, mobile sales orders, equipment information and data protection module as a business client and function of the show.

\section{Needs analysis of mobile applications on the server side}

In this paper, we study the mobile application server connection terminal at the front desk and the background server. According to different sources of background system, the data is returned to different backend server. The mobile application server management can be divided into five major functional modules as shown in figure 1.

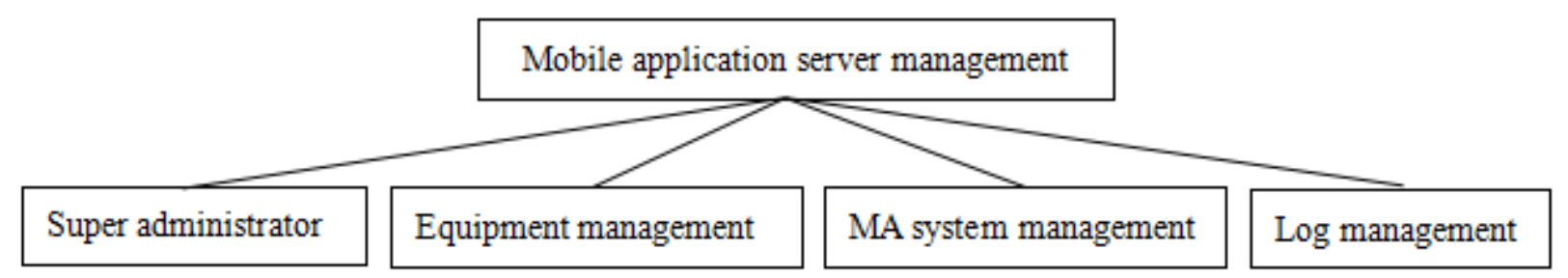

Fig 1 Mobile application server function module partition 


\section{Demand analysis of mobile client}

In this paper, we study the mobile client APP involving the scenes only do page display and business process. Conduct backend database data extraction through the mobile application server. When we have important news to inform can launch news push mechanism in the mobile client, when there is a new message, the system will automatically push to the mobile client, in order to view and handle in time. After reading news, mobile client passes backstage the new has been read. Mobile business system data processing is shown in figure 2.

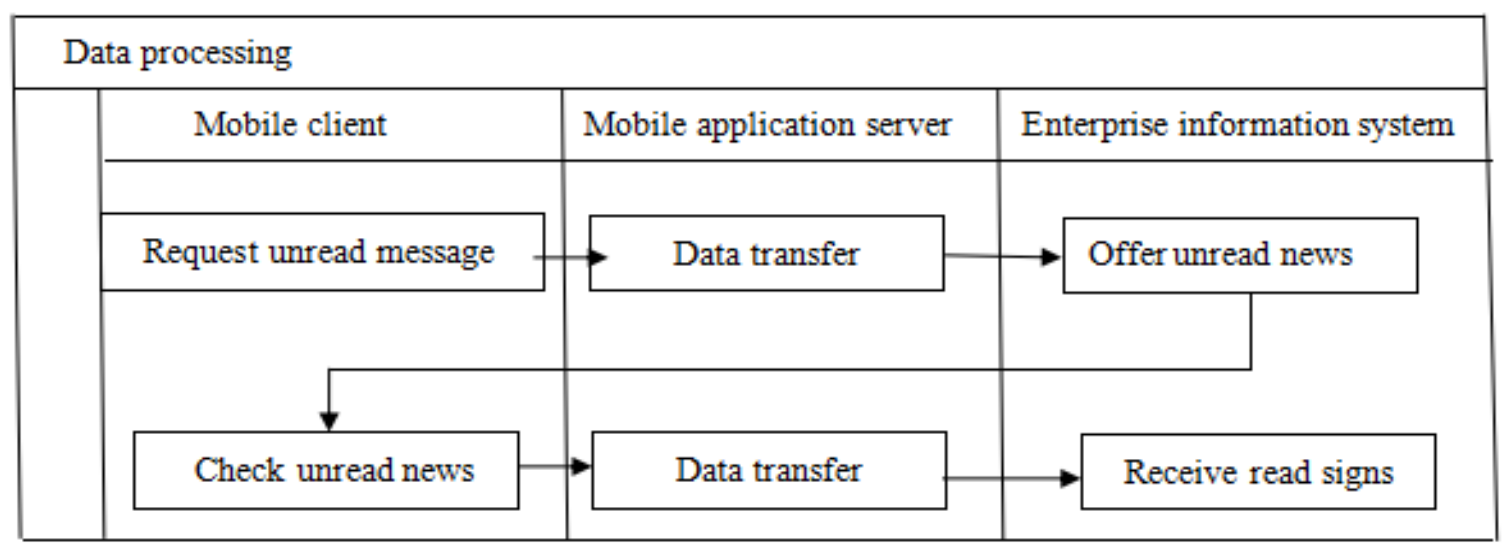

Fig 2 Mobile business system data processing schemes

\section{Related technical overview}

The use side of insurance comprehensive service system isSmart phone. It is for the insurance users all over the country, realizing comprehensive insurance services such as the long-distance cover, preservation, claims, submitted to the risk, stop protect, renewal, surrender, display and so on, which not only saves the user's time, but also make it convenient for insurance agent work, ensure the accuracy of the request, solve the bottleneck of the traditional insurance mode of operation exist, the most important thing is that it can attract more customers to pay close attention to insurance, insurance. [2]The user UI provided on the phone APP interface, beautiful and simple to use, users can easily learn thus reduce the distance between users and companies.

From the development of the overall system requirements, select the Android as a development platform, the Apache server as an intermediary web server, LAMP (Linux, Apache, MySQL, PHP) development mode for the underlying logic, the Eclipse as a development IDE implementation of Android interface development.

\section{Insurance comprehensive service system}

Insurance comprehensive service system is based on Internet. The system architecture is based on the further research of insurance business. It is on the basis of the insurance business model and absorb domestic and foreign advanced management experience. Combining with the most popular the current IT technology, comprehend insurance insurance company category at present, combine property, life insurance, reinsurance together to design and conduct large-scale systems developmentaccording to the company's actual demand analysis form the integrated service system of insurance solutions.

Insurance comprehensive service solutions require cover insurance all risks, all business process automation, informationization to provide users with comprehensive insurance company business and service, adapt to the informationization of the insurance industry, the brand market competition needs. Meet the various regions of the country's business change, meet the requirement of local, regional coverage are the core processing to provide a comprehensive solution.

Insurance comprehensive business service system realize most of the business coverage and business processes, including insurance, examination and approval, claims, submitted to the insurance and other business information processing. [3]System can implement the policy, policy administration, and other functions, thus effectively improve the efficiency of enterprise operation 
and reduce the risk of the enterprise management. Through comprehensive statistical analysis functionofthe service system, efficient, timely management and help is provided for the company's decision makers.

\section{Android platform}

Android operating system comes from the Linux, founded by Google. This platform not only provides the operating system, but also provide the UI, APPmiddleware. As an open source mobile operating system platform, Android provides a continuously updated service. The current domestic smartphones'Android version are basically all above 4.2. From the point of software architecture, Android is mainly divided into four levels, respectively is the Linux kernel, system runtime, the application architecture and applicationfrom the bottom up. As the bottom of the system, Linux kernelcan realize the interaction with the underlying hardware and provide support for the top platform. System run-time provided stock that can be used by application of the library, including $\mathrm{C} / \mathrm{C}++$ library that provide service for developers. The application architecture provides application API for developerssimplified component reuse. Application program is some basic applications based on the Android, mainly include the short message, the client browser, maps, calendar, and others based on JAVA development. The Android platform also provides simulation mobile phone interface to provide convenience for developers in the process of development.

\section{Apache application server}

Apache ranked first in the web application server usage, it is of strong universality that can be used in almost all computer platforms. Apache is an open source web application server, language is more widely used, which is commonly used by PHP, Perl, Python developersweb server, and received extensive praise. Because of Apache's advanced technology, stable performance, completely free, the characteristics of the support load balancing, it is well praised by the web developers. Web application server we are familiar with are mainly the IIS, Tomcat, Apache, IIS mainly oriented for NET platform developers, Tomcat for the JAVA platform, usuallyApache are the one we need.[4]

\section{Summary of MySQL}

MySQL is a database management system, popular with Oracle, SQL Server 2008 as the most commonly used database management system. MySQL is associated with type of database management system, the data are stored in different tables, rather than stored in large warehouse, so it can not only improve the flexibility of data but also raise the efficiency of data storage. Corresponding database languageaccess MySQL is SQL, which adopts double authorization policy, store of small volume, fast response speed, low cost features, the most important thing is that it is open source, by which it is welcomed by a vast number of software developers. MySQL is an important part of popular LAMP architecture, which uses MySQL database excellent performance to make a good development environment.

\section{Overall of design system}

\section{Overall architecture design}

\section{1) Network structure design}

Insurance company's clients access to the system via the Internet, it is safe to go through a firewall security. Insurance company employees access to the systemthrough the local area network (LAN) . Internal and external network directly deployed network gatewayto safeguard the security of system data. Data storage scheme adopts full hot standby redundancy double machine, namely equipped with two sets of database server, adopts double host method; equipped with double disk array, storage of data in the synchronized. The traditional hot Standby scheme of two, two servers generally adopts the Active/Standby, when the host fails, the Standby time can take over the host of the service. In this way, the reserved machine has been in standby state, which caused waste to a certain extent. To give full play to the efficiency of the double machine, adopt Active/Active mode, to let the standby also acts as the role of the host, share the load.

After the operation of insurance company business management system formally, the database and application system will carry out full backup automatically on the system in the last working 
day of each month. Last business day of a week is for an incremental backup database. [5]Backup files are stored in CD. When systemnot officially running, if there is update database and application system, backup files stored in CD-ROM.

\section{2) the overall structural design}

The hierarchical structure of the internal business management system is divided into system user layer, application layer, information exchange layer, logic layer and business data processing.

Users of the system layer: is made up of operations center of each employee.

Comprehensive application layer: consists of all computer operations center. Mainly refers to the show in the client's internal business management system on the page of each function module page (client), the realization of client page mainly adopts the HTML, JavaScript, and VBScript script.

Information exchange layer: used for client transmission and exchange of information between pages and services, namely through the HTML form components, under the rules of the HTTP protocol to pass the data to a network server. Logical processing layer: is composed of a network server, for the information exchange layer in the data processing and judgment, the judgment results through the SQL language after encapsulation, and the exchange of data and business data layer, so as to obtain the data repository.

Business data layer: is composed of the database server, is responsible for receiving and feedback logical processing layer to the SQL commands.

\section{Interface design}

Operation center of the internal business management system interface is divided into front-end and back-end systems. Front page doesn't have to log in, direct operation as follows: query scheduling, grades and notice; query branch contact; download all kinds of forms; search. Back-end systems require specific employees use work number and password to log in. User permissions are divided into primary and secondary. Level of authority are mainly the IT department personnel. They can undertake all the background operation, but mainly for the following operations: create registered users; to view, secondary access user management. Secondary rights are mainly administrative personnel and the national customer service center of management personnel. They are mainly for the following operations: management operations center address book publishing and management and scheduling information; issue and management; publish and manage business knowledge.

\section{Database design}

\section{1) Database structure as a whole}

Internal business management system database is mainly to keep the following three kinds of information

1. Registered users' information

2. Notices, announcements, and scheduling information of the customer service center

3. The operation center department information and staff address book

\section{2) Data table design in detail}

According to database E-R diagram, following seven tables need to be designed. Internal Login: registered user information table, Internal Notice: notice information table, Internal Announce: announcement information table, Internal Business: Business information table, InternalJobArrange: scheduling information table, Internal Call: employee directory table, Call_Dept: department information table.

\section{Summary}

Insurance information construction is the important guarantee to realize the innovation, lead insurance reform, enhance core competitiveness, and prevent the risks of operation and management. Informatization level is the important symbolto measure a insurance enterprise core competitiveness and the management level. Based on an insurance business management system implementation subject, this paper carried out the design and implementation research workon the system. In the implementation of this topic, according to the method of software engineering this 
paper completed the demand analysis, general design, detailed design, code implementation and testing, and get more skilled in business management system software work and related technology.

\section{References}

[1] Cheng Y, Kuo W, Su S. An Android system design and implementation for Telematics services[C]. //Intelligent Computing and Intelligent Systems (ICIS), 2010 IEEE International Conference on. IEEE, 2010:206 - 210.

[2] Ramlee R A, Othman M A, Leong M H, et al. Smart home system using android application[C]. Information and Communication Technology (ICoICT), 2013 International Conference of. IEEE, 2013:277 - 280.

[3] Gong Lei, Zhou Cong. Development and Research of Mobile Termination Application Based on Android [J]. Computer and Modernization, 2008(8):30 - 34.

[4] TeEn Wei, Ching Hao Mao. Android Malware Detection via a Latent Network Behavior Analysis[J]. Security and Privacy in Computing and Communications, 2012(09): 1251 - 1258.

[5] Dong, Wenli. The Research on Web Service based Network Management[J]. Website Journal. 2010(7): 849 - 852. 